\title{
XVI. Ueber flächenreiche Magnetitkrystalle aus den Alpen.
}

\author{
Von \\ Luigi Brugnatelli in München.
}

(Mit Taf. V.)

Die mineralogische Sammlung zu München besilzt in ihren reichen Suiten alpinér Mineralien auch verschiedene Vorkommnisse von flächenreichen Magnetitkrystallen, die zum Theil noch gar nicht beschrieben sind, zum Theil wenigstens Veranlassung zu einigen neuen Beobachtungen gaben, welche die kryslallographische Kenntniss dieser schönen Mineralspecies zu erweitern geeignel sind.

Die Verbreitung des Magnetites in dem Alpengebiele ist bekanntlich sehr gross, doch sind die Krystallformen desselben fast uberall sehr einfache, denn sie werden gewöbnlich durch das Oktaëder oder durch das Dodekaëder, oder auch durch die Combination beider gebildet. Nur an wenigen Fundorten treten mit diesen auch andere Formen auf. Die in dieser Beziehung wichtigsten Fundorte sind: Traversella in Piemont, Wildkreuzjoch in Pfilschthal (Tyrol), Monte Mulatto und Scalotta im Fassathal (Tyrol)*).

$\mathrm{Zu}$ diesen bis jetzl bekannten Fundorten würden nun ferner die neuen von mir untersuchten Fundorte : Rothenkopf im Zillerthal und Hollershachthal im Ober-Pinzgau, hinzukommen.

\section{Traversella.}

Die schönen Magnetite von diesem Fundorte sind schon seit lange bekannt, und das Auftreten der Formen $\{111\},\{110\}$ an denselben ist bereils

*) Ausserdem giebt C. C. von Le o oh a d d, Handbuch der Oryklognosie 1826, von Alpe della Mussa in Piemont „entkantele kautendodekaëder " an. Diese Angabe slammt vielleicht aus dem Catalogue rais. du Mus. d'hist. nat. von Borson (Turin, 1811). Strüver (N. Jahıb. 1871, 345) führl aus dem Alalhal nur einfache Dodekaëder an. 
in den ältesten Handbüchern der Mineralogie beschrieben. In einigen der letzteren sind noch andere Formen angegeben, nämlich $\{211\}$ in Quenstedt's Min. 1855 und $\{311\}$ in Hartmann, Min. 1843.

Auf Stufen, welche von diesem Fundorte stammen und aus alternirenden Schichten von Magnetit und Dolomit bestanden, beobachtete Strü ver*) neben Rhombendodekaëdern auch Krystalle der Combination $\{110\},\{321\}$ und andere, welche die letztere Form allein zeigten.

Das Muttergestein der von mir untersuchten Stufen besteht aus Dolomit und Serpentin, auf welchem der Magnetit in ringsum ausgebildeten Krystallen aufgewachsen ist und zum Theil in körnigen Massen zwischen beiden, Serpentinpartien umrandend, sich befindet. $\Lambda$ n demselben Stucke sind die Krystalle von zweierlei Habitus; ein Theil derselben zeigt die von Sırüver angeführıen Formen, zuweilen noch mit kleinen glänzenden Flächen des Oktaëders, die übrigen zeigen die einfache bekannte Combination $\{110\},\{111\}$.

Struver hal in seiner kurzen Notiz über diesen Magnetit keine Beschreibung der Flächenbeschaffenheit gegeben, was mir in diesem Falle von einiger Wichtigkeit zu sein scheint.

Wie erwähnt, sind die Flächen des Oktaëders immer glall und glänzend. Es ist bemerkenwerth, dass das Auftreten dieser Form in engem $\mathrm{Zu}-$ sammenhang steht mit der Entwickelung von $\{321\}$; indem ihre Flächen vorhanden und gut entwickelt sind, wenn $\{321\}$ fehlt oder nur sehr klein ist, während sie vollständig zurücktreten oder verschwinden, wenn die Flächen des Achlundvierziglächners grösser ausgebildet sind. Dieses Verhältniss zwischen den Flächen der zwei Formen ist so beständig und regelmässig, dass es nicht dem Zufalle zugeschrieben werden kann. Auch die Annahme, dass die Krystallisationsbedingungen, welche den Achtundvierzigfächner hervorgebracht haben, für die Entwickelung des Oktaëders nicht günstig .waren, ist in diesem Falle nicht befriedigend, da sie das regelmässige Verschwinden der letzteren Form mit dem Grösserwerden von $\{321\}$ nicht erklärt; andererseits beobachtet man, dass die Achtundvierzigflächner gerade an denjenigen Krystallen anderer Fundorte (Berg Blagodat, Hollersbachthal) gut entwickelt sind, an welchen die Hauptform das Oktaëder ist. Eine wahrscheinlichere Erklärung dieses Verhältnisses ist vielleicht zu finden in der Annahme, dass die Flächen des Achtundvierzigflächners entstanden seien, als die Krystalle schon in der Combination $\{110\},\{111\}$ ausgebildet waren. Die Ursache dieser späteren Modification der Krystalle möchte ich der Aetzung zuschreiben. Für diese Meinung spricht die constant matte und rauhe Beschaffenheit der Flächen von $\{321\}$ und die häufige Unregel-

*) Slrüver, Note mineralogichè, Alti della R. Acc. delle Scienze di Torino, 1871, 6, Sep.-Abdr. S. 19. 
mässigkeit seiner Combinalionskanten mit dem Dodekaëder. Ein Gelatinepräparat, welches ich angefertigt habe, um die Beschaffenbeit dieser Flächen besser zu untersuchen, zeigte, mil dem Mikroskop beobachtet, dass die Rauhheit der Flächen durch dreieckige Vertiefungen bewirkt wird. Wenn auch diese nicht so scharf ausgebildet waren, dass sie eine genauere Bestimmung gestattet hätten, so spricht doch diese Beschaffenheit der Flächen sehr zu Gunsten der obigen Hypothese*).

Die Dodekaëderflächen sind stets nach der langen Diagonale stark gestreift. Ein Krystall von der Combination $\{110\},\{111\}$ wurde auf dem Goniometer in Bezug auf die Reflexe gepruft und gefunden, dass beim Einstellen der Oktaëderflächen, welche gute Reflexe gaben, zugleich zahlreiche Streifen auf der Dodekaëderfläche mitspiegelten, während der Position der lelzteren nur ein unbestimmter Lichtschimmer entsprach. An derartigen Krystallen ist also die Dodekaëderfläche eine Scheinfläche, entstanden durch alternirende Ausbildung der benachbarten Oktaëderflächen. Dies ist ein Zeichen für den schalenförmigen Aufbau dieser Krystalle.

Von besonderem Interesse für die Wachsthumserscheinungen des Magnetits ist ein Krystall, welcher in Fig. 1 idealisirt abgebildet ist. Derselbe besteht aus der Combination $s\{321\}, d\{110\}$, aus deren den Hauptaxen entsprechenden Ecken diejenigen eines glatten Oktaëders hervorragen. Die letztere Form wurde also offenbar zuerst gebildet und in einem späteren Stadium fand das Krystallwachsthum nach den Flächen von $\{110\}$ statt, neben welchen, nach dem Vorigen vielleicht durch Aetzung, die Flächen des Hexakisoktaëders enstanden sind.

\section{Wildkreuzjoch.}

Am Wildkreuzjoch im Pfitschthal kommt der Magnetit in Krystallen

*) Krystalle von Rymphischwäng bei Zermatt, welche Herr Professor Groth i. J. 1883 von dort mitgebracht hat, zeigen ansserordentlich deutliche Aetzerscheinungen. Die Oklaëderflächen sind glalt, aber auf ihnen erscheinen bis $1 \mathrm{~mm}$ grosse Vertiefungen von gleichseitig dreieckiger Gestalt mit abgestumpften Ecken (Fig. 2 giebt das Bild einer Oktaèderflàche mit den betreffenden Aetzfiguren und das am Goniometer mittelst eines durch eine kleine kreisformige Oeffnung gebildeten Signales erzeugte Lichtbild); dieselben liegen umgekehrt, wie das Dreieck der Oktaëderfliche. Die Einstellung auf den hellsten Punkt des Lichtbildes ergab Werthe, die zwischen $1030^{\prime}$ und $2030^{\prime}$ schwanken und welche für die betreffende Triakisoktaederfläche der Aetziguren kein einfaches Zeichen zu bestimmen erlauben. Für die Ikositetraëderflächen war die Einstellung unmöglich. Becke beobachtete an Krystallen vom Binnenthal ebenfalls Aetzfiguren, welche aber von den obigen verschieden sind, indem bei ihnen die Aelzfiguren durch vorwiegende Ikositetraëderflächen gebildet waren und die Triakisoktaëder fehlten (s. S. 243). Bei meinen Krystallen erscheinen Triakisoktaëderflächen auch als breite und matte Aetzflàchen an den Kanten. Da, wo die Aetzung weiter vorgetreten ist und besonders nach den oktaëderecken hin, gehen diese Flächen in breite und stark gerundete, ebenlalls matle Flächen über, welche die Lage vom Hexakisoklaëder haben. 
vor, welche sich durch die Schönheit und Regelmässigkeit ihrer Flächen auszeichnen.

Von diesem Fundorte habe ich zwei prächtige Stufen untersucht, welche der hiesigen Sammlung schon seit längerer Zeit angehören.

Die Krystalle zeigen im Allgemeinen die Combination o\{111\}, $d\{110\}$, $i\{311\}, c\{100\}$ mit vorherrschendem $\{111\}$, eine Gombination, welche bereits Kenngot t*) an Krystallen vom Wildkreuzjoch beobachtet hat.

Ausser diesen Formen habe ich an einigen Krystallen auch den Pyramidenwürfel $g\{310\}$ als sehr schmale Abstumpfung der längeren Kanten des Ikositetraëders $\{311\}$ (Fig. 3) beobachtet. Zwei Messungen ergaben mir folgende Werthe :

$$
(311):(301)=17036^{\prime}, \quad 17^{\circ} 34^{\prime} ; \quad \text { Miltel 17035', }
$$

während der theoretische Werth $17033^{\prime}$ beträgt.

Die Magnetitkrystalle sitzen hier auf einem dichten Chloritschiefer und haben als Begleiter folgende Mineralien : Klinochlor, Granat, Vesuvian, Apatit, Zirkon und gelblichweissen Titanit.

Diese Mineralien wurden schon durch Hessenberg**), v on Kobell ${ }^{* * *}$ ), vom Rath t) und Kenngott (l. c.) ausfuhrlich beschrieben, so dass ich mich darauf beschränken kann zu erwähnen, dass das Auftreten des Zirkons in farblosen Krystallen und des Apatits in spitzpyramidaler Gestalt sehr charakteristisch fur diesen. Fundort ist. Nach meiner Meinung sind auch jene Begleitmineralien des Magnetits vom Widkreuzjoch dem Apatit zuzuzählen, welche von Kenngott, wenn auch zweifelhaft, als Calcit und Quarz angegeben wurden.

\section{Fassathal.}

Die Fundorle flächenreicher Magnetite im Fassathal sind Monte Mulatlo und Scalotta.

Der Apatit des ersteren Fundortes wurde zuerst von Döl ter tt) beschrieben. V. von Zepharovich $+t+$ beobachtete die Formen $\{110\}$,

*) Ke n $n$ gott, Uebersicht d. Resullate mineral. Forschungen, 1858, S: 120 und 209. S. auch: Zepha rovich, mineral. Lexicon für das Kaiserthum Oesterreich, 1872, 2, 191.

**) Hes s en berg, Mineralogische Notizen. Abhandlungen der Senkenberg. Gesellsch. 1859, 2, Sep.-Abdr. S. 13, und ebenda 1861, 4, Sep.-Abdr. S. 15.

***) von kobell, Münch. Gelehrt. Anz. 1845, S. 858 .

t) vom Rath, Mithcilungen aus der Mineralien-Samml. des H. Krantz. Pogg. An!, 108, 353 .

††) Dölter, Beiträge zur Mineralogic des Fassa-und Fleimserthals. T s che rmak's Mitth. 1877, 74 . 3, 100 .

fft) v Zepharovich, Jahresb. des Ver. Lolos in Prag, 1877, und diese Zeilschr 
$\{531\},\{311\},\{111\}$ und machte auf die Aehnlichkeit der Krystalle mit denjenigen von Achmatowsk im Ural und von Albano aufmerksam.

Die Magnetitkrystalle von Scalotta bei Predazzo, welche in der hiesigen Sammlung vertreten sind, wurden von $\mathrm{C}$ at $\mathrm{h}$ re in*) ausführlich untersucht und beschrieben. Die von ihm beobachteten Formen sind: $\{111\}$, $\{110\},\{211\},\{944\},\{522\},\{311\},\{970\},\{530\},\{531\} ;\{971\}$.

An beiden Fundorten ist die den Habitus bestimmende Form der Kryslalle das Dodekaëder.

\section{Rothenkopf im Zillerthal.}

Ueber das Vorkommen von flächenreichen Magnetitkrystallen am Rothenkopf im Zillerthal findet man keine Angaben, weder im mineralogischen Lexicon von Zepharovich, noch in Liebener und Vorha user**). Die Exemplare der hiesigen Sammlung sind durch die Mineralienhandlung von B ö h m und Wiedemann in Munchen dahin gelangt und wurden nach der Angabe des Herrn W i ede man n vor ungefähr vier Jahren gefunden. Es sind drei Stufen und ein loser prächtiger Krystall von $17 \mathrm{~mm}$ Durchmesser. An den Stufen sitzen die schönen Krystalle des Magnetits auf einem dichten Chloritschiefer und sind begleitet von derbem Apatit und ausgezeichnet dichroitischem Chlorit (Pennin). Das allgemeine Aussehen der Krystalle, deren Grösse zwischen einigen Millimetern und $2 \mathrm{~cm}$ schwankt, ist hervorgebracht durch das Vorwalten der Dodekaêderflächen, s. Fig. 4. Manchmal sind dieselben so verzerrt, dass die Orientirung einige Schwierigkeiten darbietet. führt :

Meine Beobachtungen haben zur Bestimmung folgender Formen ge-

$$
\begin{gathered}
d\{110\}, o\{111\}, c\{100\}, q\{322\}, P\{533\}, n\{211\}, i\{311\}, j\{511\}, \\
\beta\{331\}, m\{510\}, p\{722\} ?, X\{15.1 .0\} ? .
\end{gathered}
$$

Von diesen sind $\{322\},\{533\},\{311\},\{331\},\{510\}$ und die zweifelhafte $\{15.1 .0\}$ am Magnetit noch nicht beobachtet worden; $\{533\}$ und $\{15.1 .0\}$ scheinen überhaupt für das reguläre System neu zu sein.

Es wurden folgende Combinationen beobachtet (die Formen sind nach der Grösse ihrer Entwickelung geordnet):

I. $\{110\},\{111\},\{311\},\{331\},\{100\}$.

II. $\{110\},\{111\},\{311\},\{331\},\{511\},\{100\}$.

III. $\{110\},\{111\},\{311\},\{331\},\{510\},\{15.1 .0\} ?,\{100\}$.

IV. $\{110\},\{111\},\{311\},\{331\},\{511\},\{510\},\{100\}$.

V. $\{110\},\{111\},\{311\},\{331\},\{511\},\{322\},\{533\},\{211\},\{100\}$.

*) Cathrein, Ueber einige Mineralvorkommen bei Predazzo; diese Zeitschr. 8 , 219. - Neue Krystallformen tirolischer Min.; ebenda 9, 265.

**) Li ebener und Vorha user, die Mineralien Tirols. Innsbruck 1852.

Groth, Zeitschrift f. Kryatallogr. XIV. 
Fig. 4 ist das Bild der letzten Combination mit Ausnahme der Form \{533\}. Die Combination III ist die des losen Krystalles und eine Ecke desselben ist in Fig. 5 mit Ausnahme der zweifelhaften Form \{15.1.0\} abgebildet. Fig. 6 giebt das Bild einer Ecke eines Krystalles der Combination IV.

Eine besondere Aufmerksamkeit verdient die Flächenbeschaffenheit der einzelnen Formen.

Das Hexaëder ist immer sehr untergeordnet und oft wird es nur durch einen sehr schwachen Reflex am Goniometer wahrgenommen.

\{111\} ist immer gut entwickelt und von tadelloser Beschaffenheit. Vollkommen glänzend und glatt sind auch alle Ikositetraëder.

Das Ikositetraëder $\{533\}$ wurde nur an einem Krystalle beobachtet, an welchem durch ungleiche Entwickelung der Flächen des Triakisoktaëders \{331\} die durch jene Form abgestumpfte Kante zwischen Dodekaëder und Triakisoktaëder zur Ausbildung gelangte (daher ist es in die symmetrisch gezeichnete Fig. 4 nicht mit aufgenommen). Das Zeichen $\{533\}$ folgt also aus den Zonen: [111: 100], [331: 101].

Das Ikositetraëder \{722\} wurde zum ersten Male an einem Krystalle von Achmatowsk im Ural durch Pictorsky*) beobachtet. Seine Existenz an den Krystallen vom Rothenkopf ist nicht sicher. Ich habe es bei der Messung der Zone [111: 100] durch einen schwachen und unsicheren Rer flex wahrgenommen.

Die Messung ergab folgenden Werth:

beobachtet (111):(722) $=33^{0} 6^{\prime}, \quad$ berechnet $=32^{0} 44^{\prime}$.

Der Pyramidenwürfel $\{510\}$ ist immer untergeordnet; seine Flächen sind jedoch stets glatt und glänzend.

An dem oben erwähnten losen Krystalle beobachtet man in der Zone [101: 001] zwischen (105) und (001) eine gerundete, ziemlich grosse Fläche. $\Lambda \mathrm{m}$ Goniometer bemerkt man an dieser Stelle eine über ungefuhr drei Grad sich erstreckende ununterbrochene Reihe von Bildern und ganz nahe derselben einen schwachen, doch ziemlich scharfen isolirten Reflex. Eine Messung gab folgendes Resultat:

$$
(101):(h 0 l)=41012^{\prime}
$$

Aus dieser Zahl lässt sich für das betreffende Tetrakishexaëder das Zeichen $\{15.1 .0\}$ berechnen, welches den theoretischen Werth $41^{\circ} 11^{\prime}$ verlangt. Da aber auch mit einer Lupe die betreffendên Flächen nicht individualisirt werden konnten, habe ich die Form nur als zweifelhaft angenommen.

Eine höchst interessante Beschaffenheit bieten die Flächen des Dode-

*) Pi ctorsky, Zeitschr. d. d. geol. Gesellsch. 1869, 21, 489. Diese Beobaehtung wird im Jahrb. für Min. und Geol. irrthümlich $S$ a de beck zugeschrieben. 
kaëders und des Triakisoktaëders dar. Auf den ersteren ist kaum eine Spur von der charakteristischen Streifung anzutreffen, und sie sind wie die des Triakisoktaëders anscheinend glatt und manchmal recht glänzend. Beobachtet man aber die Flächen dieser Formen etwas genauer, so bemerkt man, dass sie durch zahlreiche, genau gleich orientirte Striche, welche sich bei mikrosk opischer Beobachtung als Aetzfiguren erkennen lassen, bedeckt sind. An einem Krystalle sind diese schon mit blossem Auge zu unterscheiden. Diese Aetzfiguren sind so charakteristisch für die erwähnten Flächen, dass sie an den stark verzerrten Krystallen als Anhaltspunkte für die Orientirung benutzt werden können.

Um die Gestalt dieser Aelzfiguren besser zu erkennen, habe ich von denselben Gelatinepräparate verfertigt. Die an diesen durch mikroskopische Beobachtung erhaltenen Resultate sind folgende: Die Aetzfiguren auf den Dodekaëderflächen sind in ihrer vollkommensien Ausbildung Grubchen, welche entweder die Form einer sechsflächigen oder einer vierflächigen Pyramide, die in den grösseren Figuren durch eine Basis abgestumpft wird, zeigen. Der Umriss dieser Grubchen auf den Dodekaëderflächen ist nattirlich ein sechsseitiger oder ein vierseitiger. Im ersten Falle laufen zwei Seiten (die längeren) parallel der Combinationskante $(110):(111)$, während die anderen mit diesen einen Winkel von ungefähr $103^{\circ}$ bilden; im zweiten Falle haben zwei Seiten gleiche Richtung wie bei den sechsseitigen, und die anderen zwei laufen parallel der Combinationskante (110):(100). Die kleinsten unter den Aetzfiguren bilden einfache ellipsoidische Vertiefungen, welche an den Enden der längeren Axe etwas zusammengedrückt sind. Diese Axe ist parallel der Kante (110):(111). Oft bemerkt man in der Mitte solcher Vertiefungen die vierflächige Pyramide.

Die Frage nach der Art von Flächen, welche diese Aetzfiguren bilden, kann ich leider nicht auf Grund von Beobachtungen heantworten. Ich habe zwar versucht, nach den Vorschriften von B e ck e*), wie für die Krystalle von Rymphischwäng (siehe oben), ein Lichtbild zu erzeugen, aber wegen der Kleinheit der Aetzfiguren und vielleicht aus Mangel an Erfahrung, sind meine Versuche misslungen. Ich habe jedoch beobachtet, dass die die oben beschriebenen längeren Seiten des Umrisses hervorbringenden Flächen bei der Einstellung der Oktaëderflächen schimmern. Also wüde ich sie den Flächen dieser Form zuschreiben, und umsomehr, weil diese Annahme mit den Beobachtungen von B eck e ubereinstimmt, welcher Oktaëder als primäre Aetzfläche sowohl bei künstlichen wie bei natürlichen Aetzfiguren des

*) Becke, Mehrere Untersuchungen über künstliche und natürliche Aetzfiguren der Mineralien. Tschermak's mineral. Mitth. N. F. 5, 457; 6, 237; 7, 200 (Aelzversuche an Mineralien der Magnetilgruppe); 8, 239; 9, 1 (natürliche Aetzfiguren an Pyrit, Zinkblende, Bleiglanz und Magnetit). Ref. in dieser Zeitschr. 11, 54, 273; $12,287$. 
Magnetits beobachtet hat. Durch Aetzung mit Säuren hat Becke auf den Dodekaëderflächen sechsflächige Grubchen, welche nach ihm durch zwei Oktaëder- und vier benachbarte Dodekaëderflächen gebildet werden, her-vorgebracht. Nach seinen Abbildungen aber scheinen diese Aetzfiguren mit den natürlichen vom Rothenkopf nicht übereinzustimmen. Die Aetzfiguren auf den Triakisoktaëderflächen sehen im Allgemeinen den oben beschriebenen sehr ähnlich.

Was mir der grössten Aufmerksamkeit würdig zu sein scheint, ist die Vertheilung der Aetzfiguren auf den verschiedenen Flächen der Zone zwischen Dodekaëder, Oktaëder und Würfel. Während sie auf dem Dodekaëder und Triakisoktaëder sehr zahlreich (besonders auf dem ersteren) sind, ist auf den Oktaëder- und lkositetraëderflächen keine Spur von ihnen zu bemerken, indem diese Flächen immer die grösste Regelmässigkeit zeigen. Es ist also an einem und demselben Krystalle die Angreifbarkeit der verschiedenen Flächen eine verschiedene. Diese in der Natur beobachtete Thatsache ist eine Bestätigung der von Lavizzari, Meyer, Ebner, Becke u. A. künstlich hervorgebrachten Erscheinungen. Es ist bemerkenswerth, dass, wie Becke gezeigt hat, bei der Aetzung mit Săuren die Angreifbarkeit der Dodekaëder- und Oktaëderflächen eine beinahe gleiche ist, während für das in diesem Falle in der Natur wirkende Aetzmittel die Angreifbarkeit ein Maximum auf den Dodekaëder- und ein Minimum auf den Oktaëderflächen besitzt. Gleiches Verhalten zeigen (wie aus der Beschreibung S. $239 \mathrm{zu}$ ersehen ist) auch die oben erwähnten Krystalle von Rymphischwäng.

Wie ich schon gesagt habe, sind die Formen $\{322\},\{533\},\{511\}$, $\{331\},\{510\}$ fúl den Magnetit neu. Das Zeichen $\{322\}$ folgt aus den Zonen [100:111], [313:331]. Die Messungen ergaben folgende Werthe:

$\begin{array}{lr}\text { Beobachlet: } & \text { Berechnet: } \\ (322):(111)=11021^{\prime} & 11025^{\prime} \\ (331):(322)=1857 & 1856 \\ (533):(111)=1420 & 1425 \\ (533):(101)=3054 & 3023 \\ (511):(111)=3854 & 3856 \\ (511):(511)=3132 & 3135 \\ (331):(111)=2159 & 220 \\ (110):(331)=1314 & 1316 \\ (510):(110)=3346 & 3341 \\ (510):(311)=1850 & 1854\end{array}$




\section{Oberhollersbachthal.}

Das Mineralvorkommen von Oberhollersbachthal im Pinzgau wurde bereits i. J. 1873 durch Tschermak (min.-petr. Mitth. 1873, 46) beschrieben, welcher Hessonit, Vesuvian, Diopsid, Klinochlor, Sphen, Calcit und Magnetit in glänzenden Oktaëdern beobachtete.

Die hiesige Sammlung besilzt von diesem Fundorte eine ältere Stufe, welche im Allgemeinen mit denen, die von T s chermak beschrieben worden sind, übereinstimmt. Nur fehlen Hessonit und Vesuvian, und der Magnetit erscheint nicht in Oktaëdern, sondern in Dodekaëdern mit kleinem Oktaëder. Neben diesen Flächen erscheinen unsymmetrische, matte und gestreifte Flächen, und ebenso sind die Dodekaëderflächen von mannigfaltigen Störungen der Ausbildung betroffen, namentlich von zahlreichen

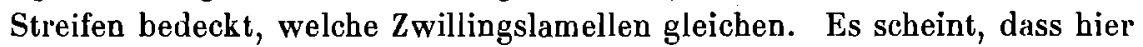
eine Störung des Wachsthums der Krystalle durch (später weggeführten) Kalkspath oder Dolomit vorliegt.

Ein ganz anderes Aussehen besitzt ein Stuck, welches Professor Groth im vorigen Jahre aus dem Pinzgau mitbrachte. Dasselbe stammt aus der Scharn im Hollersbachthal. Es besteht aus hellgrunem dichten Pistazit, dem derber Granat beigemengt ist.

Auf Drusenflächen ist vorwiegend der Epidot in kleinen glänzenden, olivengrünen, durchsichtigen Krystallen auskrystallisirt. An einem Krystalle desselben habe ich folgende Formen beobachtet:

$\{001\},\{100\},\{101\},\{201\},\{\overline{101}\},\{\overline{2} 01\},\{\overline{2} 03\},\{\overline{103}\},\{104\},\{\overline{8} .0 .11\} ?$, $\{110\},\{111\},\{111\},\{\overline{2} 33\},\{\overline{2} 21\},\{011\},\{012\}$.

Neben dem Epidot erscheint in geringer Menge schön grüngefärbter Diopsid, ganz vereinzelt sehr kleine Apatitkrystalle der Combination: $\{0001\},\{10 \bar{T} 0\},\{10 \bar{T}\},\{10 \bar{T} 2\},\{1 \mid \overline{2} 1\}$, und endlich in grösserer Menge Magnetit, zum Theil in Krystallen, die bis zu einem Centimeter Durchmesser besitzen. Das Aussehen der Krystalle ist in Fig. 7 dargestellt. Es ist die Combination des Oktaëders mit zwei Achtundvierzigflächnern, welche durch Messung als isogonal erkannt wurden.

Die beobachteten Formen sind:

$$
o\{111\}, V\{543\}, v\{13.11 .9\} \text {. }
$$

Die Hexakisoktaëder sind beide am Magnetit noch nicht beobachlet worden; $\{$ 13.11.9\} ist, so viel ich weiss, auch für das reguläre System neu.

Fast alle Krystalle zeigen die Combination der genannten drei Formen.

Die Beschaffenheit der Flächen bietet nichts Bemerkenswerthes dar. Von den beiden Achtundvierzigflächnern ist zuweilen der eine, zuweilen der andere vorherrschend; gewöhnlich aber ist $\{13.11 .9\}$ mehr entwickelt. 
Die ausgeführten Messungen sind folgende:

$\begin{array}{ccc} & \text { Beobacblet: } & \text { Berechnet: } \\ (543):(111) & =11^{2} 28^{\prime} & 11^{0} 32^{\prime} \\ (543):(534) & =1133 & 1129 \\ (13.11 .9):(111) & =826 & 839 \\ (13.9 .11):(11.9 .13)=824 & 826\end{array}$

Von den nicht alpinischen Fundorten sind in krystallographischer Hinsicht durch Flächenreichthum erwähnenswerth: Grube Zweigler bei Wildenau in Sachsen, Vesuv, Latium, Achmatowsk und Berg Blagodat im Ural.

Auf Grube Zweigler kommt der Magnetit im Braunspath vor. B reith a u p t*) beobachtete folgende Formen: $\{111\},\{100\},\{10.1 .1\},\{16.1 .1\}$.

Die Krystalle vom Vesuv wurden zuerst von Govelli und Mon ticelli in dem »Prodromo della Mineralogia Vesuviana 1825 " beschrieben. Die von ihnen angegebenen Flächen sind $\{111\},\{110\},\{100\}$ und ein nicht bestimmtes Ikositetraëder. Scacchi**) beobachtete: $\{110\},\{111\},\{311\}$, $\{553\},\{531\}$. Zu diesen Formen fugte späler Struver***i) $\{310\},\{211\}$ hinzu.

Die Untersuchungen des Magnetits von Latium gab in seiner Monographie uber die Mineralien Latiums S I r u ver $\dagger$, welcher folgende Formen beobachtete: $\{111\},\{100\},\{110\},\{211\},\{311\},\{310\},\{531\}$.

Die Krystalle von Achmalowsk und das Auftreten der Formen $\{111\}$, $\{100\},\{110\},\{311\},\{531\},\{21.7 .5\}$ an denselben sind beschrieben in den Materialien zur Mineralogie Russlands von Kokscharow t+). Pictorsky (I. c.) entdeckte noch das Ikositetraëder $\{722\}$.

Die von Jerofejew $+t+$ ) am Magnetit vom Berge Blagodat beobachteten Flächen sind: $\{111\},\{432\},\{654\}$. Die Krystalle dieses letzteren Fundortes gleichen sehr den oben beschriebenen vom Oberhollersbachthal.

Erwähnenswerth ist noch, dass bei Kraubat in Steiermark *f) und an den Krystallen von Neu-Seeland ${ }^{*} f$ ) der Wurfel als selbständige Form auftritt.

*) Breithau t, Neue Formen des tesseralen Krystallisationsystemes. Pogg. Ann. $1841,54,152$.

**) A. Scaccbi, Esame cristallografico del ferro olijisto e del ferro ossidulato. Mem. Acc. delle Scienze di Napoli 1842.

***) Diese Zeitschr. 1, 231 Z. 1 v. u.

t) Strüver, Mineralien Laliums. Diese Zeitschr. 1, 230.

$t+$ Kokscharow, Materialien etc. $\mathbf{3}, 49$.

$+f+$ J er of jew, Magneteisenerzkrystalle vom Berge Blagodat. Verhandl. der R. k. min. Gesellsch. 1882, 17 (2), 24.

*) Halle, Mineralien Steiermarks. Graz 1885, 60.

***) Gladstone, Chem. News 1, 116. 
Eine Uebersicht der bis zum Jahre 1882 bekannten Formen des Magnetits hat J e rofejew in seiner citirten Arbeit uber den Magnetit vom Berge Blagodat im Ural angegeben. Da aber seine in russischer Sprache geschriebene Abhandlung nicht leicht zur allgemeinen Verfügung sein kann, und da seitdem eine beträchtliche Anzahl neuer Formen gefunden wurden, halte ich es fur vortheilhaft, sie hier vervollständigt wiederzugeben.

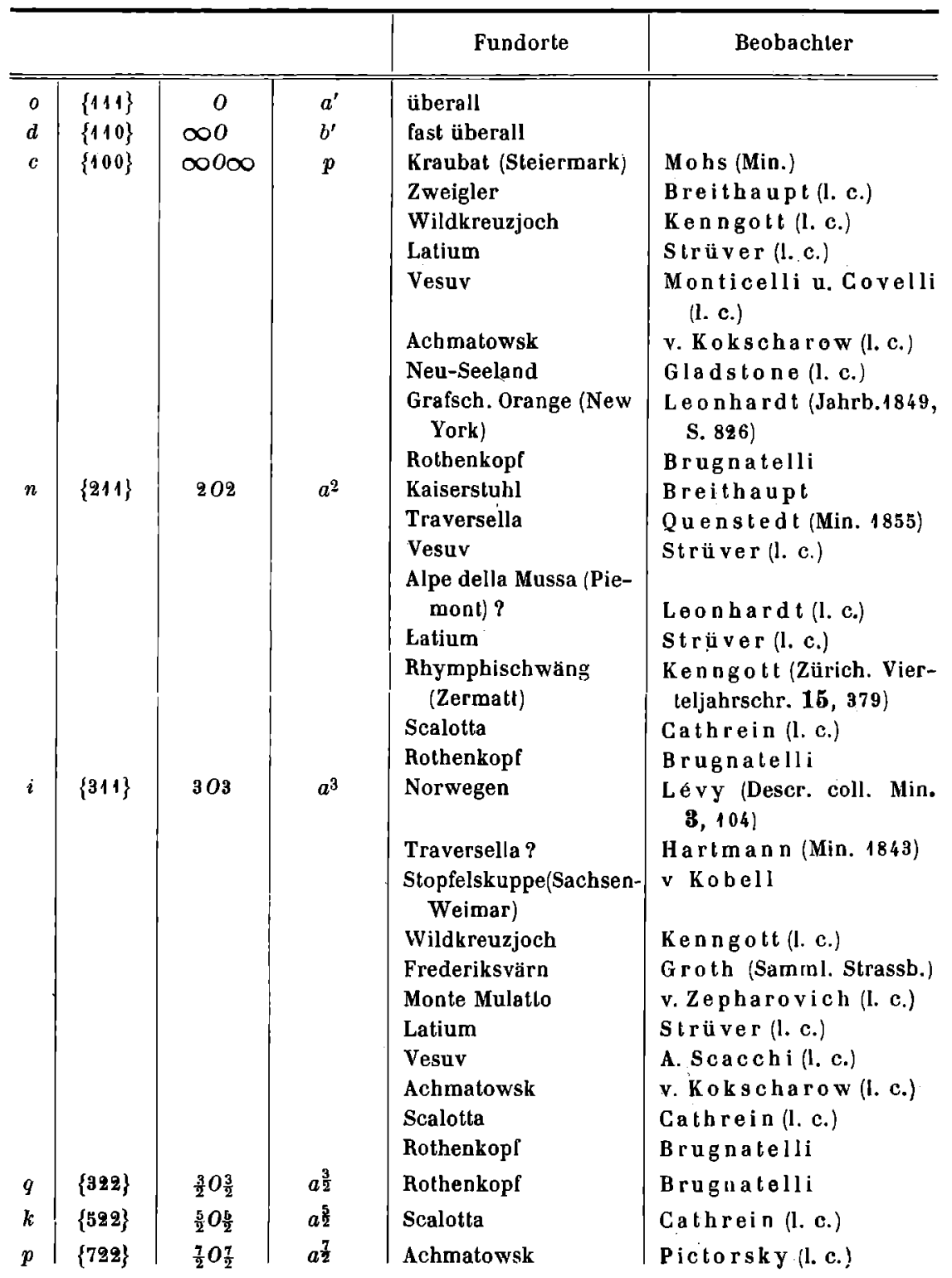




\begin{tabular}{|c|c|c|c|c|c|}
\hline & & & & Fundorte & Beobachter \\
\hline$p$ & $\{533\}$ & $\frac{5}{8} O \frac{5}{3}$ & $a_{3}^{\frac{5}{3}}$ & Rothenkopf & Brugnatelli \\
\hline$l$ & $\{944\}$ & $\frac{9}{4} 0 \frac{9}{4}$ & $a^{\frac{9}{4}}$ & Scalolta & Cathrein (l. c.) \\
\hline$j$ & $\{511\}$ & รัO5 & $a^{5}$ & Rothenkopf & Brugnatelli \\
\hline$h$ & $\{611\}$ & 606 & $a^{6}$ & $?$ & Descloizeaux (Manuel) \\
\hline$N$ & $\{10.1 .1\}$ & 10040 & $a^{10}$ & Zweigler & Breithaupt(l. c.) \\
\hline$M$ & $\{16.1 .1\}$ & 16096 & $a^{16}$ & Zweigler & Breithaupt (I. c.) \\
\hline$\alpha$ & $\{221\}$ & 20 & $a^{\frac{1}{2}}$ & Zillerthal (?) & Hartmann (Min. 1829) \\
\hline$\beta$ & $\{331\}$ & 30 & $a^{\frac{1}{3}}$ & Rothenkopf & Brugnatelli \\
\hline$\gamma$ & $\{553\}$ & $\frac{5}{3} O$ & $a^{\frac{3}{5}}$ & Vesuv & A. Scacchi (I. c.) \\
\hline$c$ & $\{210\}$ & $\infty 0 z$ & $b^{2}$ & $?$ & $?$ \\
\hline$g$ & $\{310\}$ & $\infty 03$ & $b^{3}$ & $\begin{array}{l}\text { Vesuv(Monte Soma) } \\
\text { Lalium } \\
\text { Wildkreuzjoch } \\
\text { Roihenkoof }\end{array}$ & $\begin{array}{l}\text { Strüver (l. c.) } \\
\text { Strüver (l. c.) } \\
\text { Brugnatelli }\end{array}$ \\
\hline$f$ & $\{530\}$ & $\infty 0 \frac{5}{3}$ & $b^{5}$ & Scalotla & Cathrein (l. c.) \\
\hline$e$ & $\cdot\{970\}$ & $\infty 0_{9}^{9}$ & $b^{\frac{9}{7}}$ & Scalotta & Cathrein (1. c.) \\
\hline$x$ & $\{15.1 .0\}$ & $\infty 015$ & $b^{15}$ & Rothenkopf (?) & Brugnatelli \\
\hline$s$ & $\{321\}$ & $30 \frac{3}{2}$ & $b^{1} b^{\frac{1}{2}} b \frac{1}{3}$ & $\begin{array}{r}? \\
\text { Traversella }\end{array}$ & $\begin{array}{l}\text { Brooke und Miller } \\
\text { (Min. 1852) } \\
\text { Strüver (l. c.) }\end{array}$ \\
\hline $\boldsymbol{R}$ & $\{432\}$ & $20 \frac{4}{3}$ & $b \frac{1}{2} b \frac{1}{3} b \frac{1}{4}$ & Berg Blagodat & Jerofejew (1. c.) \\
\hline $\boldsymbol{V}$ & $\{543\}$ & $\frac{5}{3} O \frac{5}{4}$ & $b \frac{1}{3} b \frac{1}{4} b \frac{1}{6}$ & Oberhollerbachsthal & Brugnatelli \\
\hline$r$ & $\{654\}$ & $\frac{3}{2} O_{\frac{6}{5}}^{6}$ & $b \frac{1}{4} b \frac{1}{5} b \frac{1}{6}$ & Berg Blagodat & J erof ejew (l, c.) \\
\hline$v$ & $\{13.11 .9\}$ & $\frac{13}{y} 0_{1}^{1 \frac{3}{2}}$ & $b \frac{1}{9} b \frac{1}{11} b r^{\frac{1}{3}}$ & Oberhollersbachthal & Brugnatelli \\
\hline$z$ & $\{531\}$ & ธ०० $\frac{5}{3}$. & $b^{1} b^{\frac{1}{3}} b^{\frac{1}{5}}$ & $\begin{array}{l}\text { Vesuv } \\
\text { Achmatowsk } \\
\text { Latium } \\
\text { Monle Mulatto } \\
\text { Scalotta }\end{array}$ & $\begin{array}{l}\text { A. Scacchi (l. c.) } \\
\text { v. Kokscharow (l. c.) } \\
\text { Strüver (l. c.) } \\
\text { v. Zepharovich (l. c.) } \\
\text { Cathrein (l. c.) }\end{array}$ \\
\hline$y$ & $\{971\}$ & 909 & $b^{1} b^{\frac{1}{7}} b^{\frac{1}{4}}$ & Scalotta & Cathrein (l.c.) \\
\hline$x$ & $\{21.7 .5\}$ & ${ }_{5}^{21} 03$ & $b \frac{1}{5} b \frac{1}{7} b \frac{1}{2}$ & Achmatowsk & v. Kokscharow (l. c.) \\
\hline
\end{tabular}

Die Tabelle enthält also 31 Formen, nämlich, ausser den drei einfachen Formen des regulären Systemes, elf Ikositetraëder, drei Triakisoktaëder, fünf festgestellte und ein unsicherer Pyramidenwürfel, endlich acht Hexakisok taëder.

Von diesen Formen sind, so viel ich weiss :

$\{722\},\{533\},\{944\},\{553\},\{530\},\{970\},\{432\},\{654\},\{13.11 .9\},\{971\}$, $\{21.7 .5\},\{15.1 .0\}$

bis jetzt nur am Magnetit beobachtet worden.

Es ist sehr auffallend, dass es mir nicht möglich war, fur die Formen $\{210\},\{221\}$, welche auch in den älteren Handbüchern der Mineralogie, wie 
in denjenigen von $\mathrm{Naumann}(1846)$ (in diesem ist \{221\} angegeben), von B rooke und Miller (1852), Z ippe (1859) etc. als bekannte Formen des Magnetits angegeben werden, den ersten Beobachter und den Fundort, an welchen sie beobachtet worden sind, zu finden.

$\{210\}$ scheint aber von Mohs herzurühren (s. Naumann, Reine und angewandte Krystallographie 1830, 1, 246) *).

Von allgemein krystallographischem Interesse ist es, dass alle Achtundvierzigflächner des Magnetit, mit Ausnahme von $\{971\}$ und $\{21.7 .5\}$, welche verhältnissmässig complicirte Zeichen haben, zu jener Classe von Hexakisoktaëdern gehören, deren Grenzformen $\{111\},\{210\}$ sind, und welche wegen der Eigenschaft, dass die sechs an den hexaëdrischen Ecken zusammenstossenden Kanten gleiche Winkel besitzen, von $\mathrm{Na}$ u ma $\mathrm{n} \mathrm{n}$ »isogonale " genannt worden sind. Bekanntlich ist das allgemeine Zeichen solcher Hexakisoktaëder :

nach Naumann $m O \frac{2 m}{m+1}$, nach Miller $\left(h \cdot \frac{h+l}{2} \cdot l\right)$.

Aus Fig. 8, der Projection sämmtlicher Formen des Magnetit, geht hervor, dass dieselben sich besonders um das Oktaëder häufen und wenige derselben in der Nähe des Würfels und Dodekaëders liegen. Die erstere Form ist auch dadurch als das wichtigste Glied der Formenreihe ausgezeichnet, dass sie stets die beste Flächenbeschaffenheit zeigı und ihr auch die Spaltbarkeit der Krystalle parallel geht.

*) Im Handbuch der Mineralogie von Hartmann (1829) ist beim Magnetit \{221\} abgebildet und als vom Zillerthal beobachtete Form angegeben. 